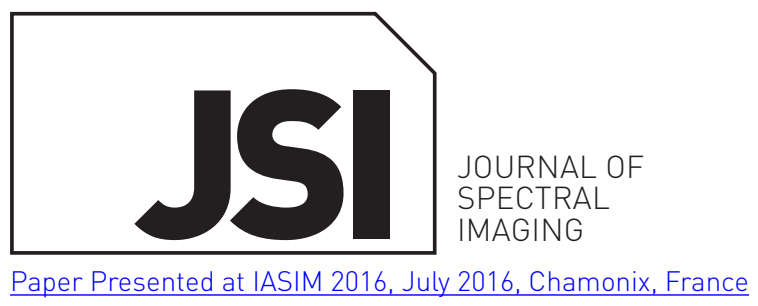

\title{
openaccess
}

\section{Use of infrared hyperspectral imaging as an aid for paint identification}

\author{
A. Polak, ${ }^{a, b,{ }^{*}}$ T. Kelman, ${ }^{a}$ P. Murray, ${ }^{a}$ S. Marshall, ${ }^{a}$ D.J.M. Stothard, ${ }^{b}$ N. Eastaugh ${ }^{c}$ and F. Eastaugh ${ }^{c}$ \\ ${ }^{a}$ Centre for Signal \& Image Processing, University of Strathclyde, 204 George Street, Glasgow, G1 1XW, United Kingdom \\ ${ }^{b}$ Fraunhofer Centre for Applied Photonics, Fraunhofer UK Research Ltd, 99 George Street, Glasgow, G1 1RD, United Kingdom \\ ${ }^{c}$ Art Analysis and Research Ltd, 162-164 Abbey Street, London, SE1 2AN, United Kingdom. E-mail: adam.polak@strath.ac.uk
}

\begin{abstract}
Art authentication is a complicated process that often requires the extensive study of high value objects. Although a series of nondestructive techniques is already available for art scientists, new techniques, extending current possibilities, are still required. In this paper, the use of a novel mid-infrared tunable imager is proposed as an active hyperspectral imaging system for art work analysis. The system provides access to a range of wavelengths in the electromagnetic spectrum (2500-3750 nm) which are otherwise difficult to access using conventional hyperspectral imaging (HSI) equipment. The use of such a tool could be beneficial if applied to the paint classification problem and could help analysts map the diversity of pigments within a given painting. The performance of this tool is demonstrated and compared with a conventional, off-the-shelf HSI system operating in the near infrared spectral region (900-1700 nm). Various challenges associated with laser-based imaging are demonstrated and solutions to these challenges as well as the results of applying classification algorithms to datasets captured using both HSI systems are presented. While the conventional HSI system provides data in which more pigments can be accurately classified, the result of applying the proposed laser-based imaging system demonstrates the validity of this technique for application in art authentication tasks.
\end{abstract}

Keywords: hyperspectral imaging (HSI), infrared, laser imaging, optical parametric oscillator (OPO), art work authentication, classification, support vector machine (SVM)

\section{Introduction}

The art market is a highly sophisticated one where the value of a particular piece is often predetermined by its authorship and, critically, its authenticity. Unfortunately, with a high demand for various art works, the market is often supplied with counterfeit creations. As a result, authentication of the art work, particularly for high value pieces, is an important aspect of this market. Common practice in the art world is connoisseurship-an evaluation of the piece based on non-scientific expertise. However, when this process is not supported by scientific tests, it often provides limited proof of the assessment. Various scientific methods may be applied to art analysis and authentication and these, in parallel with the knowledge of art historians and connoisseurs, have the potential to empower the evaluation with substantial evidence about the authenticity of the piece. ${ }^{1}$ Many of the scientific techniques which can be employed require the removal of small samples of material from the painting, which is highly undesirable, especially for assessing high value objects. Therefore, there is a clear need for new, non-destructive scientific practices that can support the analysis and evaluation of art work in an efficient and effective way. Several techniques, such as X-ray fluorescence and Fourier transform infrared (FT-IR) or Raman spectroscopy, $^{2,3}$ are already available and used by art historians. In this paper, hyperspectral imaging (HSI) systems operating in the infrared (IR) spectral region are presented as an additional non-destructive testing technique that can be used to support art authentication studies. To this end, the performance of an active, laser-based, mid-infrared (mid-IR) hyperspectral 
imager is compared with a passive near infrared (NIR) hyperspectral camera (see below for more details on both systems) when applied to the challenge of pigment and paint classification.

$\mathrm{HSI}$ is already recognised as a valuable technique in the art world and it has been successfully used for mapping of different paints in the art work, ${ }^{4,5}$ material identification ${ }^{6-9}$ and analysis of the artists' techniques. ${ }^{10,11}$ Recently, an application of mid-IR imaging device for art work analysis was also demonstrated. ${ }^{12}$ In this work, studies were conducted using a subset of the mid-IR spectral region which was demonstrated to provide meaningful information for art scientists.

The mid-IR imager used in this work was originally developed as a device for stand-off detection of gases and explosive materials, where only the presence or absence of a compound would be judged based on the detected reflected energy at a specific wavelength. This paper demonstrates a novel application of this system, where a full hyperspectral data set is acquired and chemometric algorithms are applied for its analysis. Techniques for accurate image acquisition and the subsequent application of chemometrics to extract useful information are the main focus of this study. The results of the classification techniques used provide a quantitative overview of the performance of both systems operating individually and give an indication of the pigment recognition accuracy that could be achieved.

\section{Materials and methods Hyperspectral equipment}

The HSI system under evaluation in this study is a Firefly IR Imager (M Squared Lasers). This is an active, laser-based system, providing scanned point-by-point illumination to the object (in this case paintings) under study. The device operates mainly in the mid-IR region (2500-3750 nm), however, it can also acquire images from a narrow band of the NIR spectral range (1490-1850 nm). Firefly's vertically polarised laser source operates in a pulsed regime with $150 \mathrm{kHz}$ repetition rate, $<10 \mathrm{~ns}$ pulse duration and average powers of $P_{\text {av_NIR }}=140 \pm 30 \mathrm{~mW}$ and $P_{\text {av_midlR }}=90 \pm 40 \mathrm{~mW}$ in the NIR and mid-IR, respectively. It should be noted that both average powers quoted are variable as a function of wavelength. The image acquisition process is based on single point detection of reflected laser energy. The nominal laser beam radius at the output port is $2 \mathrm{~mm}\left(1 / \mathrm{e}^{2}\right.$ intensity) with $1.5 \mathrm{mrad}$ half angle beam divergence. A spatial scan is made possible by two, galvanometer-mounted, gold mirrors which deflect the illuminating laser beam and direct the collected, reflected energy back to the single-element IR photovoltaic detector. Firefly is able to capture images up to maximum pixel count of $51 \times 512$ pixels (that may have various spatial resolutions, depending on the beam deflection settings as well as the distance between the imager and the objectl) and three lower options are available: $64 \times 64,128 \times 128$ and $256 \times 256$ pixels. However, due to the sinusoidal movement pattern of the vertical galvanometer, nearly $12 \%$ at the bottom and at the top of the image are spatially distorted. As a result, in our work, these regions of each image are cropped before data analysis. A hyperspectral image can be captured by performing the sequential collection of spatial images across the spectral range that is accessible by the device.

Access to the mid-IR is granted by the fact that the Firefly IR Imager uses intracavity optical parametric oscillator (ICOPO) technology, which transforms the radiation of a $1064 \mathrm{~nm}$ pump laser into two beams at longer wavelengths, corresponding to the aforementioned spectral range of this system. ${ }^{13}$ When the pump photon passes the non-linear crystal (fan grated, periodically poled lithium niobate, PPLN), where the parametric process takes place, its energy is converted into two lower energy photons called signal and idler. The summed energy of the signal and idler photons is equal to the energy of the pump photon. The split of the energy between signal and idler depends on the position of the crystal relative to the pump laser and therefore its translation across the pump beam allows spectral tuning of the imager. While the technology is highly innovative, the requirement for a physical translation of the crystal accompanied by the time required for image collection at each wavelength makes the acquisition of a hyperspectral cube time consuming. In fact, the total acquisition time is determined by the required spectral and spatial resolution of the hyperspectral data cube to be acquired. Since the down-converted spectral region is continuous across the entire accessible bandwidth, the spectral resolution of the hyperspectral data cube is determined by the step size of the crystal translation. As a result, the spectral tuning may be as fine as $0.1 \mathrm{~nm}$. However, due to the finite linewidth of the illuminating laser $\left(\sim 5 \mathrm{~cm}^{-1}\right)$, in this work the hyperspectral data cubes (hypercubes) were acquired with a spectral resolution of $6 \mathrm{~nm}$. It should be noted that the option for the spectral tuning of this imager gives it the ability to perform as a hyperspectral, multispectral or single band imager depending on user requirements.

In the sections below, the performance of the Firefly IR imager is compared with that of a Red Eye 1.7 (Inno-spec $\mathrm{GmbH}$ ) passive hyperspectral camera. This system employs the widely used pushbroom data acquisition method ${ }^{14}$ that is common with this kind of hardware. The Red Eye 1.7 system uses a transparent grating which provides 256 spectral image bands across the NIR operating range (900-1700 nm). As a passive system, the Red Eye 1.7 requires external illumination and off-the-shelf halogen lamps were used during imaging, to ensure a sufficient amount of infrared illumination.

\section{Data acquisition}

Image acquisition with the Red Eye 1.7 passive imaging system was performed following a procedure that is typical for such systems and provided high quality data. A Zolix KSA 11-200S4N linear translation stage was used to provide relative movement between scanned pieces and the detector. This is required by pushbroom imaging systems which capture images in a line scan fashion. By providing sufficient and even illumination 

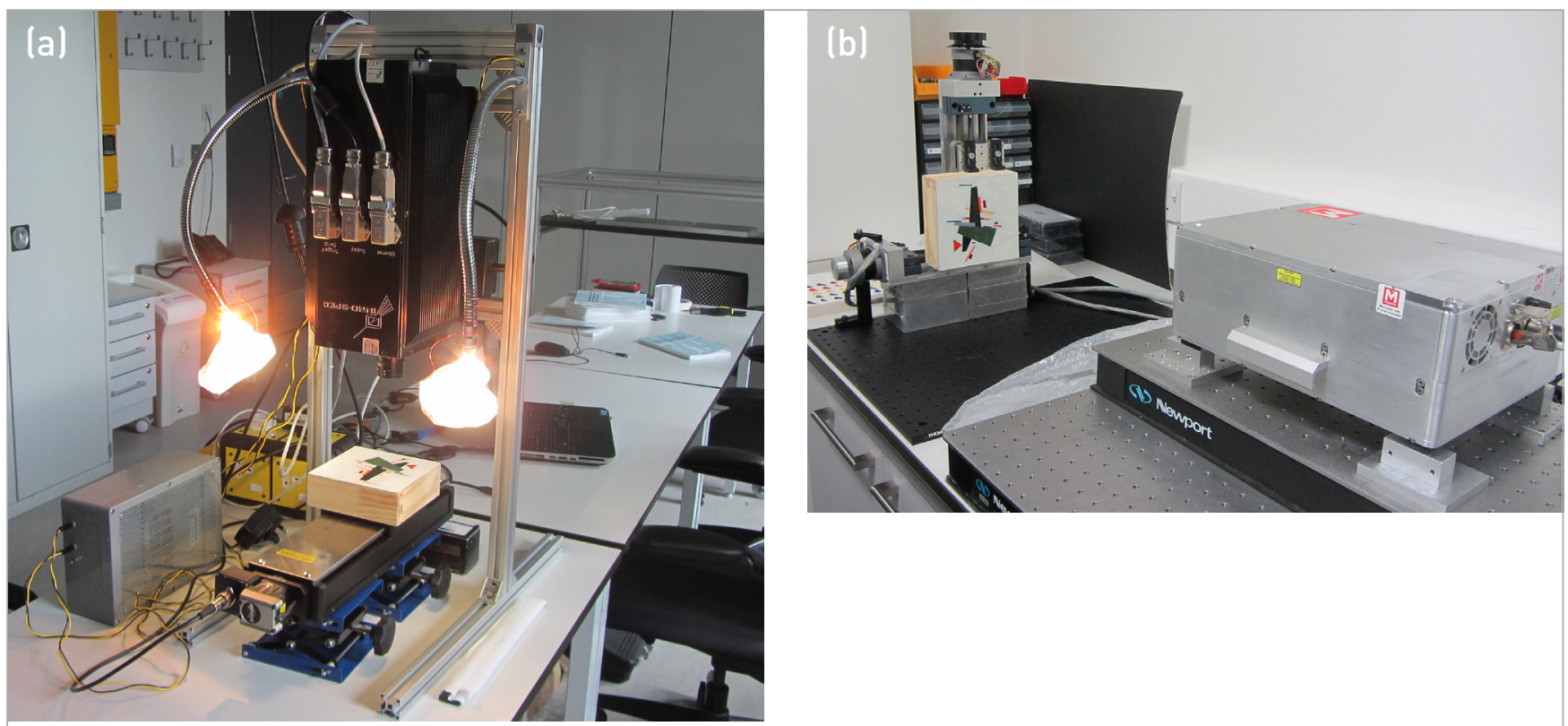

Figure 1. Illustration of the scanning set-up for Red Eye 1.7 camera (a) and Firefly IR Imager (b).

from halogen lamps and the scanning mechanism, the hyperspectral data cubes were acquired for a number of different paintings. Before acquiring the data, reflectance calibration of the system was performed using separate white and dark references. A Spectralon ${ }^{15}$ tile was used as a white calibration target and a dark reference was obtained by obscuring the camera objective with an opaque black cap. As the data acquisition software provided with the Red Eye 1.7 camera incorporates the traditional calibration procedure, the captured data was saved directly in the reflectance format and no further calibration or correction was required. Figure 1 (a) illustrates the set-up of the full scanning system which incorporates the Red Eye 1.7 camera.

Although simpler in principle, imaging with the laser-based Firefly IR device proved to be a more complicated process. As a result, the initial captured data demonstrated several features, inherent to the underlying physics driving this imager, which make direct application of chemometrics difficult. The main obstacle is that the spatial intensity distribution across the image is not uniform. Unfortunately, due to the deflection of the illuminating beam, the collection efficiency of the reflected energy varies with respect to the angle of incidence of the beam on the object. This phenomenon results in a varying reflectance response of objects which are spectrally identical and should therefore produce the same spectrum regardless of spatial location. Figure 2 illustrates a single band image (at $3200 \mathrm{~nm}$ ) of a test paint grid (see the Building the spectral library section), where the whole spatial area was captured with one scan. The background across the image is covered with one paint and, in the absence of the aforementioned issue, should have the same intensity across the whole image. To enhance visibility of this phenomenon with the naked eye, during acquisition of the image presented in Figure 2, the gain of the detector preamplifier was reduced, resulting in higher

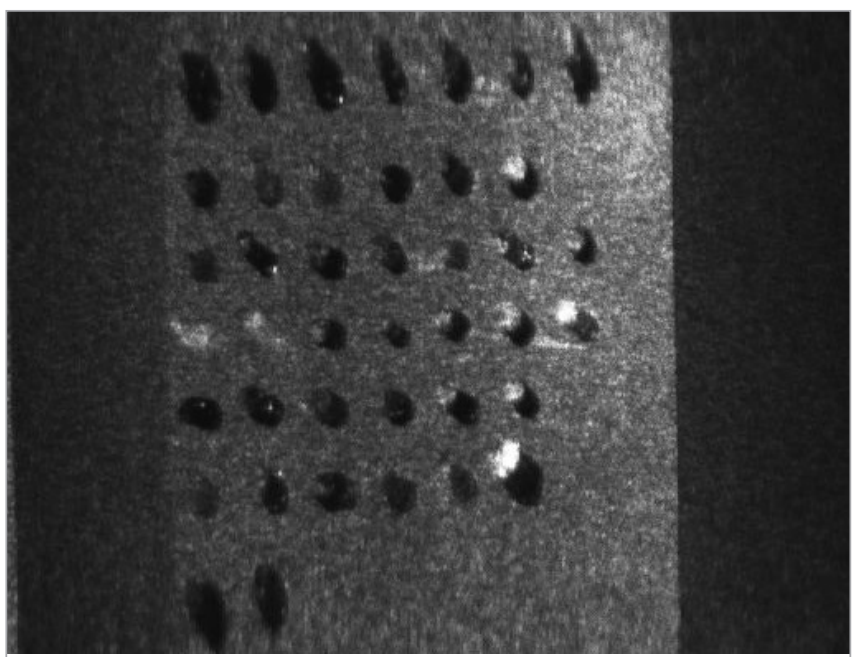

Figure 2. Single band image (at $3200 \mathrm{~nm}$ ) of the test paint grid illustrating spatial intensity variation of collected reflected illumination.

contrast between the areas of varying intensity. The imaging set-up and laser scanning incidence angles were analysed further to provide us with a better understanding of this problem. Figure 3 provides a schematic diagram of the imager position shown with respect to the image plane. The schematic illustrates the approximate laser beam incidence angles during the scanning process. This image also demonstrates the off-normal incidence of the illumination beam on the object placed parallel to the housing of the imager. For roughened surfaces, diffuse reflections are best observed when the scanning beam is at a near-normal angle of incidence with respect to the object. According to the bidirectional reflectance distribution, with an increasing angle of incidence, the main intensity of the reflected radiation will be directed away 


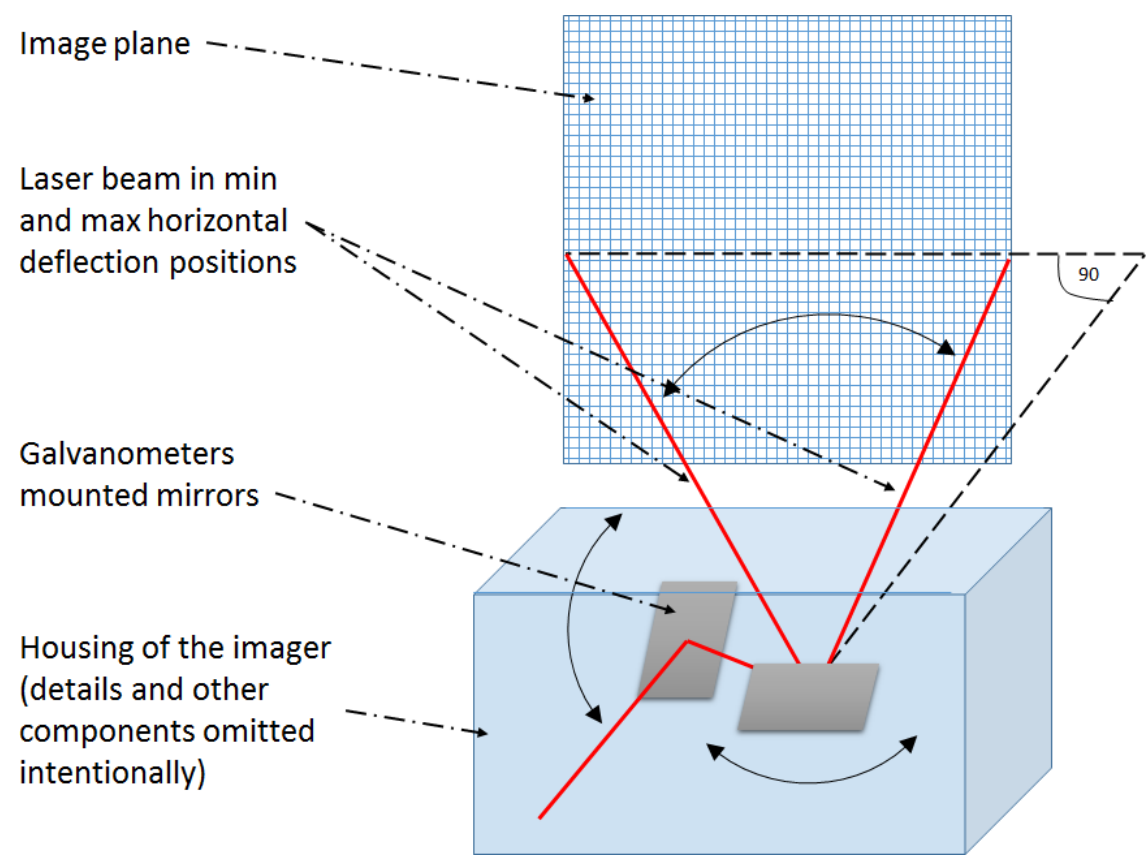

Figure 3. Simplified diagram of the minimum and maximum beam positions at one horizontal plane during the image scan.

from the detector. ${ }^{16}$ This phenomenon accurately describes the observed behaviour of the Firefly IR Imager.

Due to the off-normal incidence of the laser beam when imaging the painting placed parallel to the front of the Imager housing, it was decided to image the painting at three different orientations, as illustrated in Figure 4. This allowed us to experiment with and verify the impact that the incidence angles of the scanning beam have on the intensity of the reflected radiation that is collected. The test paint grid was first placed parallel to the imager housing, then $+45^{\circ}$ and finally at $-45^{\circ}$ from the normal parallel position. Since it was also observed that the intensity variation is stronger in the longer wavelength range, this test was performed in the NIR operating band of the system where this phenomenon is weaker. Any undesired artefacts observed in images at these wavelengths are expected to be amplified in the mid-IR range.

The results shown in Figure 4 confirm the expected behaviour and are consistent with the bidirectional reflectance distribution studies-increasing the angle of incidence results in a reduction in the amount of radiation that is reflected back to the detector.

Since the Firefly is effectively a closed, COTS system, there are a limited number of options available to address this issue. The first approach that was considered aimed to provide a uniform reference target (similar to the white tile approach) that could be imaged periodically and used for normalisation and correction of all images captured using the same set-up. However, during trials it was discovered that spatial uniformity in the image data is highly dependent on the imaging wavelength as well as the type and surface roughness of the materialls) which make up the object being imaged.
This initial approach was therefore unsuccessful, since the data could not be corrected using a single reference target. Following a number of unsuccessful trials using various materials specially machined for consistency and dimensioned to be larger than the paintings under study, this approach was abandoned.

Eventually, the most appropriate solution involved scanning each painting in small individual spatial segments of $50 \times 75 \mathrm{~mm}$ (the size of these segments was derived empirically) to produce multiple small images which were subsequently stitched together to produce a full-size image for each wavelength. This procedure limited the deflection of the scanning beam and thus improved the spatial intensity distribution across the scanned area. The result of this data acquisition method is illustrated in Figure 5(c). The scanning system including the Firefly IR Imager and the painting placed on a linear $x-y$ translation stage, which allowed accurate and repeatable image portioning into the small blocks described, is illustrated in Figure 1 (b).

Other artefacts in the data captured using the Firefly IR imaging system were caused by specular reflections from the paints and due to the three-dimensional nature of the surface of the paintings. Any curvature on the paint surface resulted in reflections of the incident beam in various directions-thus causing significant variations in the reflected energy detected by the imager. Similarly, in some places, specular reflections occurred and the energy was reflected directly back to the detector leading to saturation. This too had significant impact on the measured spectrum of the affected paints. Having no control over the structure of the paintings, it was decided to include all these artefacts into the same class of specific paint when applying statistical classification techniques to 


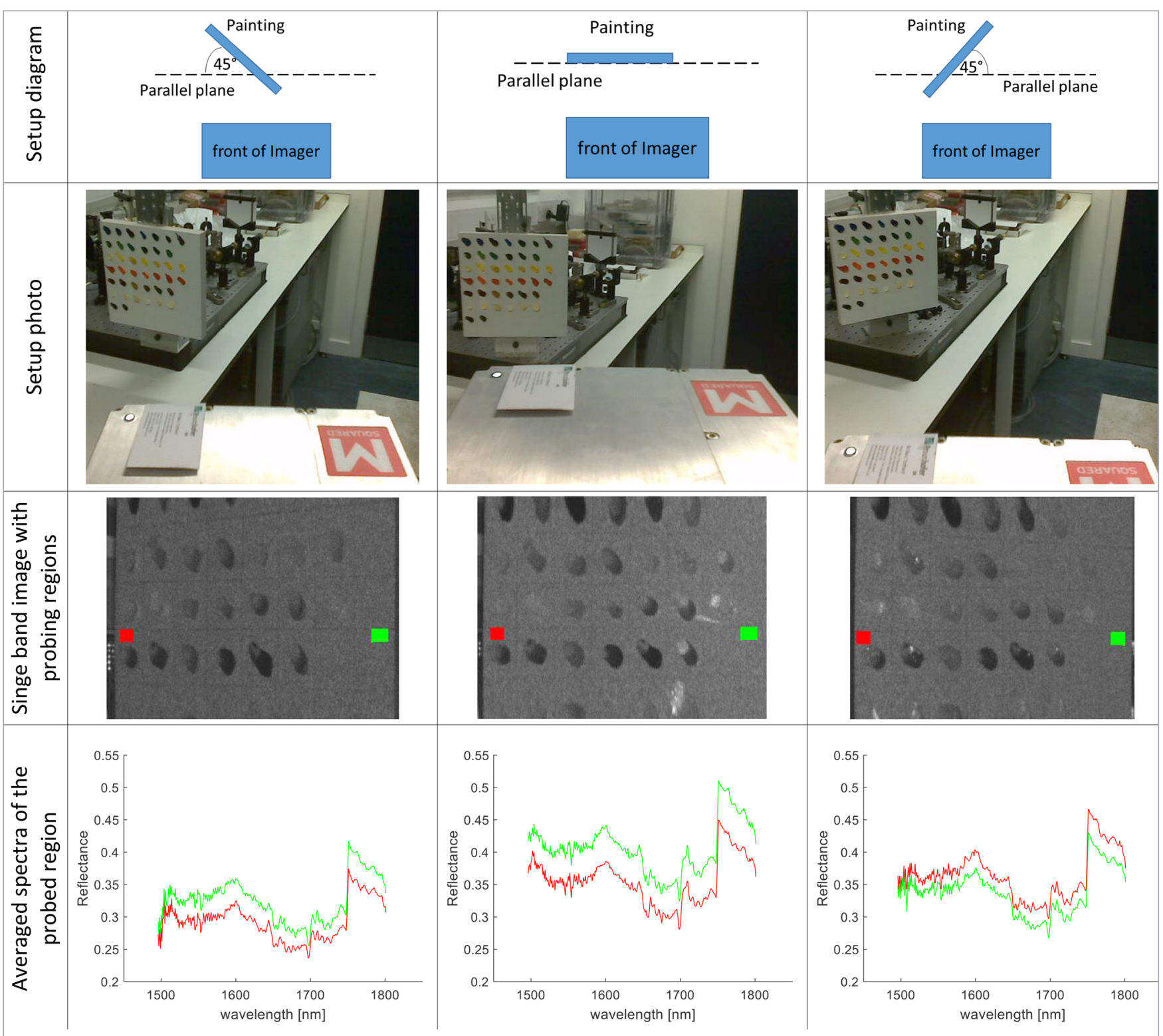

Figure 4. Results of the test for the impact of painting position on the intensity variation across the image.

automatically recognise paints based on their spectral signatures. Last but not least, the image quality acquired by the Firefly IR Imager is affected by the speckle issue (caused by the coherence of the illumination radiation); however, no mitigation techniques for the speckle phenomenon were applied during this work.

\section{Spectral pre-processing}

Data from the passive system was acquired as calibrated reflectance values. However, due to technicalities of the hardware structure, it was impossible to calibrate data from the Firefly IR Imager in a similar manner. Therefore, before any further processing, the 8-bit data of hyperspectral data cubes acquired using the Firefly IR system were rescaled to the range [0-1] by dividing each intensity by the maximum value (255).

\section{Building the spectral library}

The main goal of this work was to verify the use and application of the aforementioned hyperspectral imagers to the challenge of paint classification. The first step was therefore to create a spectral library of paints which were subsequently used as training data for the proposed automated classification process. A grid canvas with a selection of 41 paint samples was prepared [see Figures 5(a) and 5(b)] based on paints purchased from a number of specialist artists' colourmen. Suppliers were selected with particular reference to those offering high quality ranges that include ostensibly "historically appropriate" materials (Michael Harding; Rublev; Blockx). The grid canvas was imaged using both HSI systems [see single band intensity images in Figures 5(c) and 5(d)] and, for each paint, a spectral signature was extracted from the hyperspectral data cube and used to train a classifier. Averaged spectral profiles of 


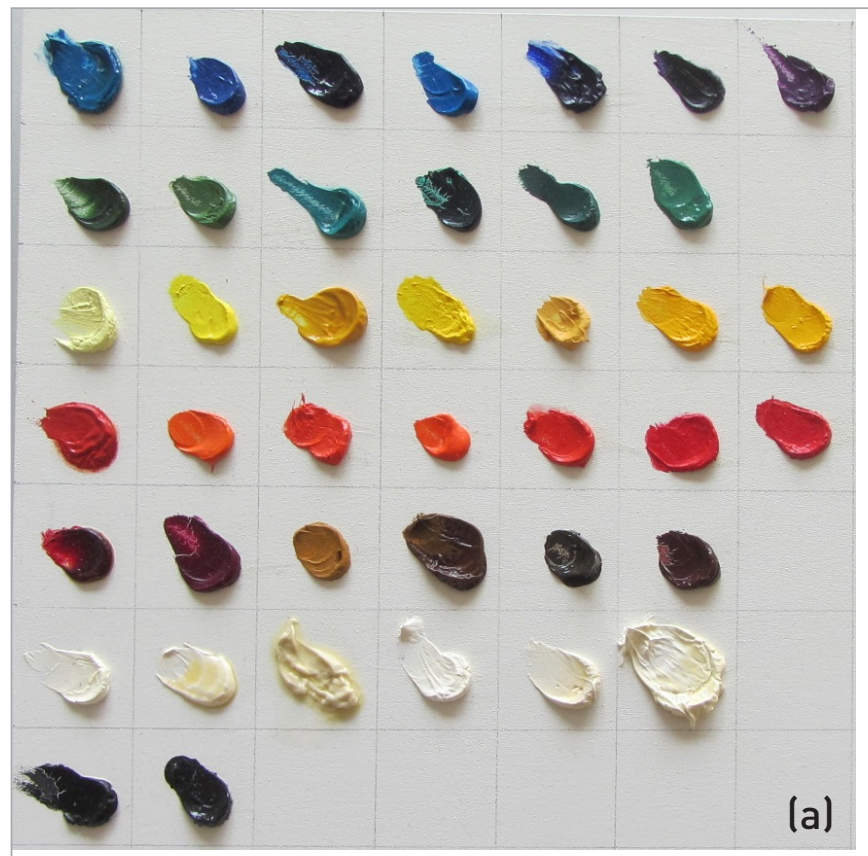

\begin{tabular}{|c|c|c|c|c|c|c|}
\hline $\begin{array}{l}\text { Cerulean } \\
\text { blue }\end{array}$ & $\begin{array}{l}\text { Cobalt } \\
\text { blue }\end{array}$ & $\begin{array}{c}\text { Phthalo } \\
\text { blue lake }\end{array}$ & $\begin{array}{c}\text { Manganese } \\
\text { blue }\end{array}$ & $\begin{array}{l}\text { Ultramarine } \\
\text { blue }\end{array}$ & $\begin{array}{c}\text { Dioxazine } \\
\text { violet }\end{array}$ & $\begin{array}{c}\text { Manganese } \\
\text { violet }\end{array}$ \\
\hline Terre vert & $\begin{array}{l}\text { Chrome } \\
\text { oxide } \\
\text { green }\end{array}$ & $\begin{array}{c}\text { Cobalt } \\
\text { turquoise }\end{array}$ & $\begin{array}{l}\text { Phthalo } \\
\text { green lake }\end{array}$ & Viridian & $\begin{array}{l}\text { Chrome } \\
\text { green }\end{array}$ & \\
\hline $\begin{array}{l}\text { Lemon } \\
\text { yellow }\end{array}$ & $\begin{array}{l}\text { Bright } \\
\text { yellow } \\
\text { lake }\end{array}$ & Aureolin & $\begin{array}{l}\text { Yellow } \\
\text { lake }\end{array}$ & $\begin{array}{l}\text { Naples } \\
\text { yellow }\end{array}$ & $\begin{array}{l}\text { Cadmium } \\
\text { gold } \\
\text { yellow }\end{array}$ & $\begin{array}{l}\text { Chrome } \\
\text { yellow }\end{array}$ \\
\hline Vermilion & Minium & $\begin{array}{c}\text { Orange } \\
\text { molybdate }\end{array}$ & $\begin{array}{c}\text { Pyrrolo } \\
\text { vermilion }\end{array}$ & $\begin{array}{l}\text { Napthol } \\
\text { red }\end{array}$ & $\begin{array}{l}\text { Scarlet } \\
\text { lake }\end{array}$ & $\begin{array}{c}\text { Cadmium } \\
\text { red }\end{array}$ \\
\hline $\begin{array}{l}\text { Alizarin } \\
\text { crimson }\end{array}$ & Magenta & $\begin{array}{l}\text { French } \\
\text { yellow } \\
\text { ochre }\end{array}$ & $\begin{array}{l}\text { Raw } \\
\text { sienna }\end{array}$ & $\begin{array}{l}\text { Raw } \\
\text { umber }\end{array}$ & $\begin{array}{c}\text { Transparent } \\
\text { oxide red }\end{array}$ & \\
\hline $\begin{array}{l}\text { Cremnitz } \\
\text { white }\end{array}$ & $\begin{array}{l}\text { Flake } \\
\text { white }\end{array}$ & $\begin{array}{l}\text { Barite } \\
\text { white }\end{array}$ & $\begin{array}{l}\text { Zinc } \\
\text { white }\end{array}$ & $\begin{array}{l}\text { Flemish } \\
\text { white }\end{array}$ & $\begin{array}{l}\text { Titanium } \\
\text { white \#3 }\end{array}$ & \\
\hline $\begin{array}{l}\text { Ivory } \\
\text { black }\end{array}$ & $\begin{array}{l}\text { Lamp } \\
\text { black }\end{array}$ & & & & & (b) \\
\hline
\end{tabular}

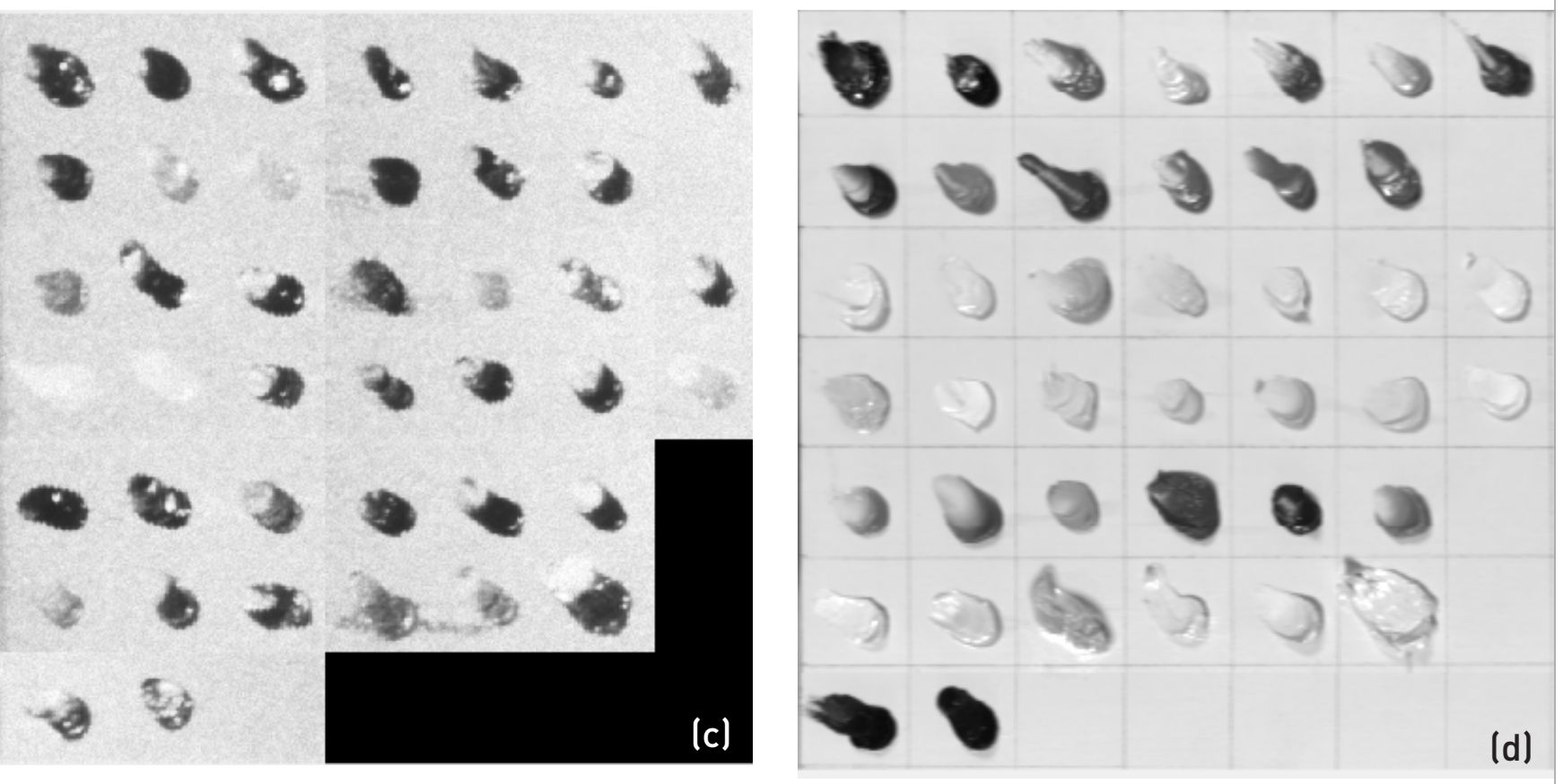

Figure 5. Illustration of the grid canvas used as training data, imaged with a RGB camera (a), description of the pigments used in the grid canvas (b), Firefly IR imager-single band at $3200 \mathrm{~nm}$ (c) and Red Eye 1.7-single band at $1200 \mathrm{~nm}$ (d).

three different paints selected for the purposes of example are shown in Figure 6. It should be noted that the spectral signatures of paints in the NIR-calibrated dataset obtained using the Red Eye 1.7 have been compared with and were shown to be similar to the corresponding spectra available in the database of pigment spectra. ${ }^{17}$

\section{Classification}

A dual-stage classification approach was proposed to automatically detect paints in the hyperspectral data cubes. For each classification problem, a subset of three paints was first selected manually based on the colour information in order to limit the training set to the compact set of likely candidate paints. Then, a detail, pixel-by-pixel, spectral classification was performed using the hyperspectral data captured for these candidate paints from both imaging systems. Although the first stage in this classification approach could be easily automated based on RGB information or visible range hyperspectral data, it was performed manually in this work to quickly test the feasibility of the proposed hyperspectral 


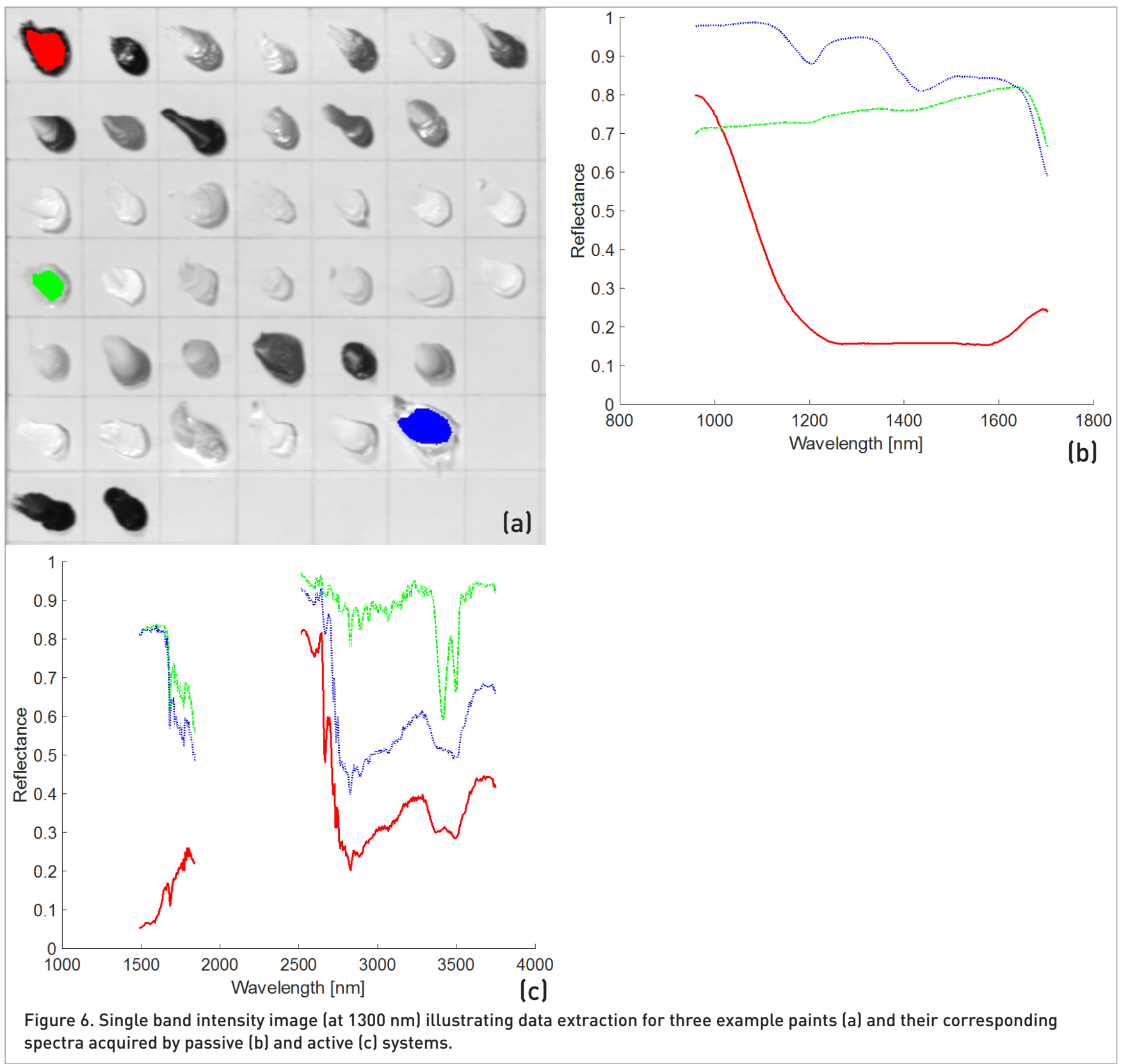

image analysis approach. A support vector machine (SVM) was chosen for this second stage classification. SVM is a robust machine learning algorithm which is widely used and consistently provides good classification accuracy. ${ }^{18,19}$ The algorithm used is based on the LIBSVM ${ }^{20}$ package and the multiclass problem was carried out using a "one-against-one" approach.

\section{Results}

In addition to the grid canvas used for training purposes, we also realised a bespoke painting which was a pastiche of a Suprematist work by Kazimir Malevich that was generated with a subset of paints available in the training set shown in Figure 2(a). This painting was also imaged with both avail- able systems using the exact same approach as outlined in the Materials and methods section. To avoid redundant processing, a set of masks were used to select regions of interest in the image. This had the added advantage of limiting each classification run to only homogeneous areas in the painting which had been created using a paint of the same colour. Classification was applied to all masked regions in turn before a majority voting scheme was executed to allocate each region to a single class corresponding to the paint used there. Figure 7 illustrates one example of all the steps from this process.

Repeating the classification process for the whole painting allowed a map of the results to be generated for both HSI data sets, and these are shown next to the ground truth image in Figure 8. The regions labeled as misclassified come from the 


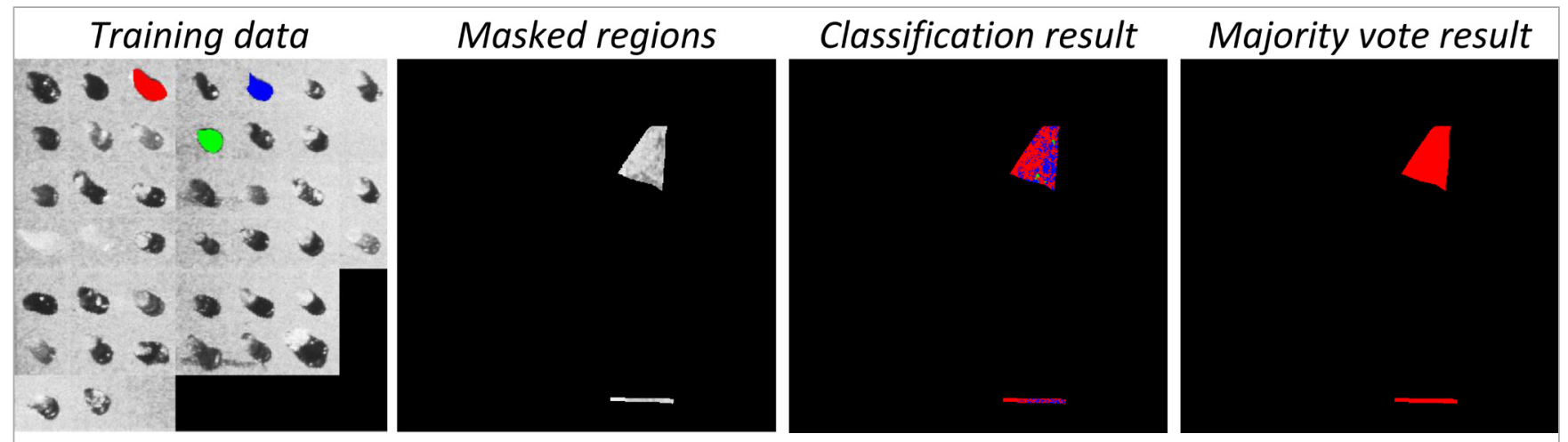

Figure 7. Illustration of the analysis approach for pigment classification on the tested painting. For each colour on the painting, a subset of training paints was chosen and classification was performed on the masked area of the painting corresponding to this colour. Classification resulted in per-pixel classification of the selected area and majority vote was drawn for these regions resulting in selection of a single pigment corresponding to one colour (demonstration based on Firefly IR Imager data).

\begin{tabular}{|c|}
\hline Phthalo blue lake \\
\hline Terre vert \\
\hline Cobalt turquoise \\
\hline Chrome yellow \\
\hline Minium \\
\hline Napthol red \\
\hline Alizarin crimson \\
\hline Titanium white \#3 \\
\hline Ivory black \\
\hline not available \\
\hline MISCLASSIFIED \\
\hline
\end{tabular}

(a)
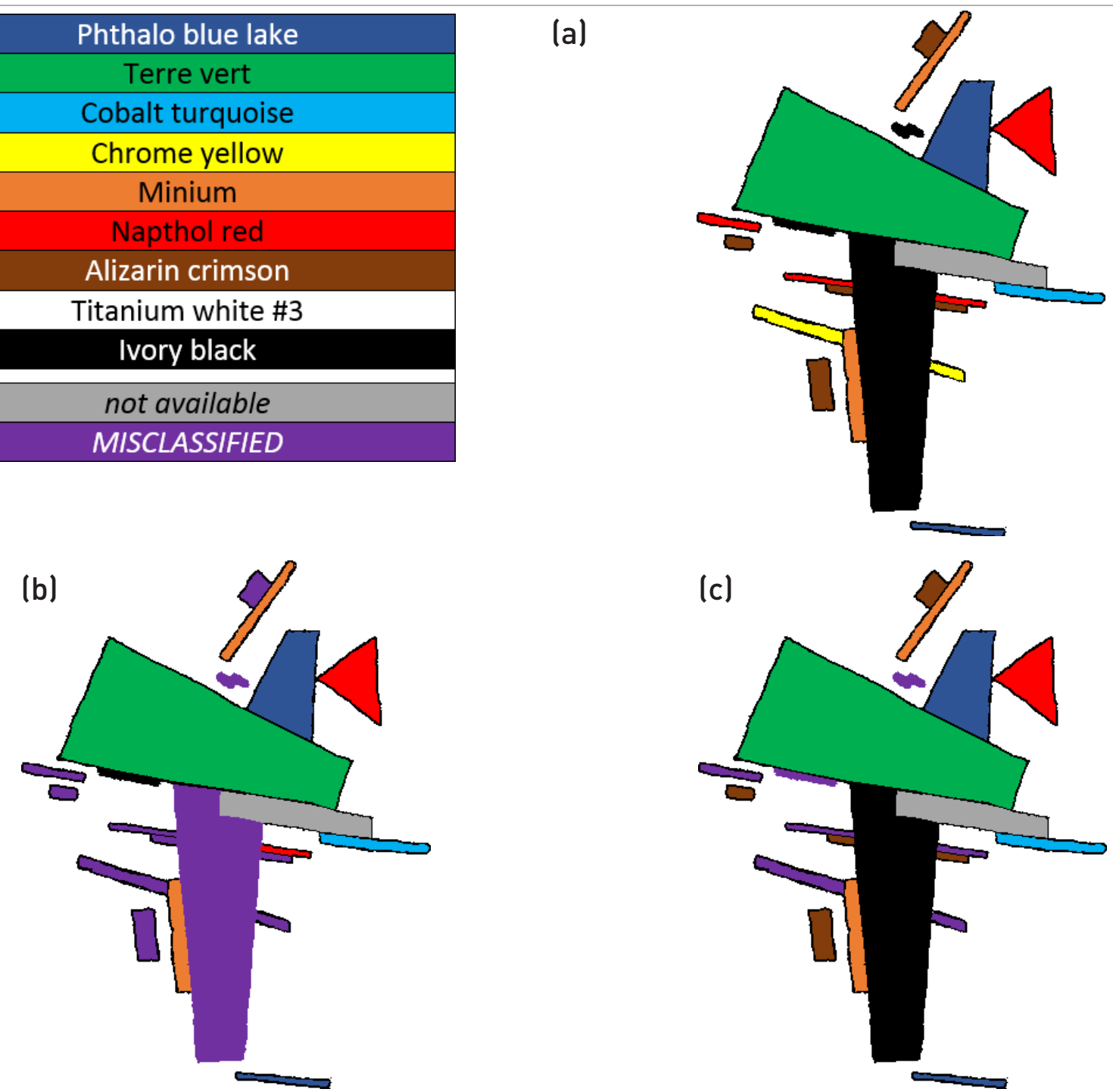

Figure 8. Graphical illustration of the bespoke painting ground truth with colour coding of paints (a) and classification results for Firefly IR Imager (b) and Red Eye 1.7 Imager (c). 


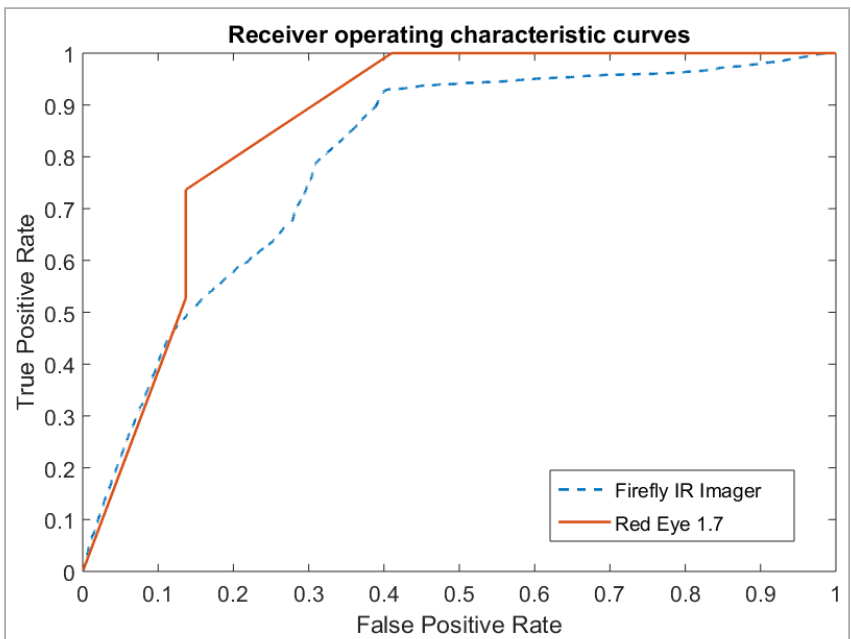

Figure 9. Example of ROC curves for both tested HSI sensors.

classification result where majority vote resulted in assigning a class different than the one from ground truth.

The automated analysis and classification of the dataset captured using the Firefly IR Imager resulted in the correct classification of $67 \%$ of pigments (six out of nine) that were present in the painting. The data set captured using the Red Eye 1.7 imager resulted in $78 \%$ (seven out of nine) of the paints being correctly identified.

For each pixel in the masked, analysed region of the image, a unique class label was assigned from the subset of three candidate paints. The percentage of correctly classified pixels varied significantly between different regions in spite of the fact that each region was known to have been painted using a unique paint from the library. The misclassification problem was most likely due to the spectral similarity of the candidate paints or as a result of the imaging artefacts discussed in the Data acquisition section. Figure 9 shows the receiver operating characteristic (ROC) curves for both HSI sensors. The ROC curves were generated by applying the classifier to the data from both HSI systems and performing a binary comparison with the output and a set of manually generated ground truth data. It is clear that the performance of the classification using the data from the passive system is better than the active one. This reduced accuracy in the active system can be explained by the presence of the aforementioned imaging artefacts (as discussed in the Data acquisition section) which are present in the data set.

\section{Conclusions}

This study has demonstrated the application of chemometric techniques to identify pigments and paints in hyperspectral images of a bespoke painting. It is shown that infrared hyperspectral imaging is a powerful, non-destructive tool able to support the art authentication process. Furthermore, such a system could be used for pigment classification and hence automatic mapping of paints within a painting to help guide the sampling process while potentially helping to reduce the amount of pigment sampling required.

The data in this work was acquired using a state-of-the-art, laser-based hyperspectral imager and the results of analysing these images have been compared to those obtained when analysing data captured using a conventional hyperspectral camera. It is shown that there are various challenges associated with capturing wide-area hyperspectral images using active laser-based imaging systems such as the Firefly IR. These occur as a result of non-uniform back-scatter reflectance and specular reflections which affect the nature of the spectral signatures observed. To address these challenges, we have demonstrated a workable solution and have shown that it is possible to successfully apply signal processing algorithms to the resulting data set to achieve accurate classification in most cases.

The comparison of the classification results from both systems was in favour of the conventional hyperspectral camera; however, the score was very close between both systems. Although the Firefly IR Imager is a very powerful tool and enables stand-off detection in the mid-IR region, the image quality and time of hyperspectral data acquisition would have to be improved to compete with conventional designs of hyperspectral cameras. On the other hand, the spectral region covered by this system is rarely available in other systems. Therefore, when the spectral signature is required in this bandwidth, e.g. for the detection of hydrocarbons or other substances with strong spectral features in this region, the Firefly IR Imager is able to collect a valid hyperspectral data set where other conventional HSI systems cannot.

\section{Acknowledgements}

This study was carried out within the framework of an Intelligent Hyperspectral Imaging (INHERIt) Project financed by Innovate UK, Technology Strategy Board in collaboration with Department of Electronic \& Electrical Engineering, University of Strathclyde, (Glasgow, UK), Fraunhofer UK (Glasgow, UK), M Squared Lasers (Glasgow, UK) and Art Analysis \& Research (London, UK). This project was also supported by an Industrial Fellowship from the Royal Commission for the Exhibition of 1851.

\section{References}

1. N. Eastaugh and J. Nadolny, "Science for the art market: a buyer's guide", ArtBanc Market Intelligence No. 7, 33-37 (April 2007).

2. A. Adriaens, "COST Action G8: Non-destructive analysis and testing of museum objects", in Benefits of NonDestructive Analytical Techniques for Conservation, Ed by A. Adriaens, C. Degrigny and J.A. Cassar (2005).

3. G.D. Smith, J.F. Hamm, D.A. Kushel and C.E. Rogge, "What's wrong with this picture? The technical analysis 
of a known forgery", in Collaborative Endeavors in the Chemical Analysis of Art and Cultural Heritage Materials. American Chemical Society, pp. 1-21 (2012). doi: http:// dx.doi.org/10.1021/bk-2012-1103.ch001

4. K.A. Dooley, S. Lomax, J.G. Zeibel, C. Miliani, P. Ricciardi, A. Hoenigswald, M. Loew and J.K. Delaney, "Mapping of egg yolk and animal skin glue paint binders in Early Renaissance paintings using near infrared reflectance imaging spectroscopy", Analyst 138, 4838-4848 (2013). doi: http://dx.doi.org/10.1039/c3an00926b

5. H. Liang, "Advances in multispectral and hyperspectral imaging for archaeology and art conservation," Appl. Phys. A 106, 309-323 (2012). doi: http://dx.doi. org/10.1007/s00339-011-6689-1

6. J.A. Toque, Y. Sakatoku and A. Ide-Ektessabi, "Pigment identification by analytical imaging using multispectral images", Conference Paper in Proceedings / International Conference on Image Processing (December 2009). doi: http://dx.doi.org/10.1109/icip.2009.5414508

7. A. Casini, F. Lotti, M. Picollo, L. Stefani and E. Buzzegoli, "Image spectroscopy mapping technique for non-invasive analysis of paintings", Stud. Conserv. 44, 39-48 (1999). doi: http://dx.doi.org/10.2307/1506694

8. J.K. Delaney, J.G. Zeibel, M. Thoury, R. Littleton, M. Palmer, K.M. Morales, E.R.d.l. Rie and A. Hoenigswald, "Visible and infrared imaging spectroscopy of Picasso's Harlequin Musician: mapping and identification of artist materials in situ", Appl. Spectrosc. 64, 584-594 (2010). doi: http://dx.doi.org/10.1366/000370210791414443

9. J.K. Delaney, M. Thoury, J.G. Zeibel, P. Ricciardi, K.M. Morales and K.A. Dooley, "Visible and infrared imaging spectroscopy of paintings and improved reflectography," Heritage Science 4(6), 1-10 (2016). doi: http://dx.doi. org/10.1186/s40494-016-0075-4

10. A. Cardeira, S. Longelin, S. Costa, A. Candeias, M. Carvalho and M. Manso, "Multi-analytical characterisation of D'Aprés Cormon by José Veloso Salgado", Nucl. Instrum. Meth. Phys. Res. B 331, 271-274 (2014). doi: http://dx.doi.org/10.1016/j.nimb.2013.12.032

11. M. Attas, E. Cloutis, C. Collins, D. Goltz, C. Majzels, J.R. Mansfield and H.H. Mantsch, "Near-infrared spectroscopic imaging in art conservation: investigation of drawing constituents," J. Cultur. Heritage 4, 127-136 (2003). doi: http://dx.doi.org/10.1016/S12962074(03)00024-4

12. F. Rosi, C. Miliani, R. Braun, R. Harig, D. Sali, B.G. Brunetti and A. Sgamellotti, "Noninvasive analysis of paintings by mid-infrared hyperspectral imaging", Angew. Chem. Int. Edit. 52, 5258-5261 (2013). doi: http:// dx.doi.org/10.1002/anie.201209929

13. D.J.M. Stothard, M.H. Dunn and C.F. Rae, “Hyperspectral imaging of gases with a continuous-wave pumpenhanced optical parametric oscillator", Optics Express 12, 947-955 (2004). doi: http://dx.doi.org/10.1364/ OPEX.12.000947

14. H. Grahn, Techniques and Applications of Hyperspectral Image Analysis. Wiley, Chichester (2007). doi: http:// dx.doi.org/10.1002/9780470010884

15. Labsphere, Inc., Spectralon Diffuse Reflectance Standards. Available online: https://www.labsphere.com/site/ assets/files/2628/spectralon diffuse reflectance standards.pdf [Accessed 5 September 2016]

16. K.E. Torrance and E.M. Sparrow, "Theory for off-specular reflection from roughened surfaces", J. Opt. Soc. Am. 57, 1105-1114 (1967). doi: http://dx.doi.org/10.1364/ JOSA.57.001105

17. M. Picollo, G. Nasilissi, C. Cucci, L. Stefani and M. Tsukada, Ultraviolet, Visible and Near Infrared Reflectance Spectra of Modern Pictorial Materials in the 200-2500 nm Range. Available online: http://drs.ifac.cnr.it/ [Accessed 5 September 2016].

18. C.-W. Hsu, C.-C. Chang and C.-J. Lin, A Practical Guide to Support Vector Classification. National Taiwan University, Taiwan (2010).

19. N. Cristianini and J. Shawe-Taylor, An Introduction to Support Vector Machines and Other Kernel-Based Learning Methods. Cambridge University Press, Cambridge (2000). doi: http://dx.doi.org/10.1017/CB09780511801389

20. C.-C. Chang and C.-J. Lin, "LIBSVM : a library for support vector machines", ACM Trans. Intell. Syst. Technol. 2, 27:1-27:27 (2011) 